\title{
Factores que inciden en la conducción en la Escuela de Medicina en la Bluefields Indian and Caribbean University
}

\author{
Nereyda Jackson Rodríguez ${ }^{1}$
}

\section{RESUMEN}

En este artículo se exponen algunos factores que inciden en la Escuela de Medicina en el marco del fortalecimiento de la educación superior, teniendo en cuenta que la conducción es un aspecto amplio e integral, dinámico y flexible, máximo cuando se trabaja con personas interactivas comunicativas. El objetivo fue determinar los factores que inciden en la conducción de la Escuela de Medicina en la Bluefields Indian and Caribbean University BICU, Región Autónoma de la Costa Caribe Sur de Nicaragua. Según el nivel inicial del conocimiento es explorativa, con un enfoque cualitativo, es descriptiva, de corte transversal. Las técnicas utilizadas en recopilación y procesamiento de información la constituyó la matriz FODA (fortaleza, oportunidades debilidades y amenaza), así como la aplicación de un cuestionario con cuatro dimensiones: liderazgo, motivación, reciprocidad o compromiso y participación. Los resultados obtenidos en la investigación pueden ser de utilidad para mejorar la conducción de la escuela de medicina.

Palabras claves: fortaleza, oportunidad, debilidades, amenaza, clima organizacional.

Recibido: 12 de julio de 2017

Aceptado: 27 de noviembre de 2017

1 Docente de la Bluefield Indian and Caribbean University BICU. Doctoranda del programa Gestión y Calidad de la Investigación Científica. FAREM Estelí-UNAN Managua. Correo electrónico: nerey_jackson@yahoo.es 


\title{
Elements that influence in the leading of the Medical school at the Bluefields Indian and Caribbean University
}

\begin{abstract}
This paper presents some elements that influence in the Medical School within the context of strengthening higher education, taking into account that leading is a broad and integral, dynamic and flexible aspect, maximum when working with interactive communicative people. The objective of this paper was to determine the elements that influence in leading the School of Medicine at Bluefields Indian and Caribbean University BICU, Autonomous Region of the South Caribbean Coast of Nicaragua. According to the initial level of knowledge is explorative, with a qualitative approach, is descriptive, cross-sectional. The techniques used for data collection were constituted by the SWOT matrix (strength, opportunities, weaknesses and threats), as well as the application of a questionnaire with four dimensions: leadership, motivation, reciprocity or commitment and participation. The results obtained in the research can be useful to improve the leading of the medical school.
\end{abstract}

Keywords: strength, opportunity, weaknesses, threat, organizational climate. 


\section{INTRODUCCIÓN}

En el marco del Fortalecimiento de la docencia y la investigación en tema de calidad de la educación superior, se presenta esta investigación que responde a la siguiente interrogante ¿Cuáles son las fortalezas, oportunidades, debilidades, amenazas, y clima organizacional que presenta la Escuela de Medicina? Es una investigación no experimental, cuyo objetivo fue determinar los factores que inciden en la conducción de la escuela de medicina en la Bluefields Indian and Caribbean University (BICU) primer trimestre del 2017. Se aplicó la técnica FODA (fortalezas, oportunidades, debilidades y amenazas) y un cuestionario. Los resultados se enfocan en la determinación de fortalezas, oportunidades, debilidades y amenazas del objeto de estudio y sobre clima organizacional desde un ámbito exploratorio. Cash J, (2017), afirma que desde el año 2008, se apertura la carrera de medicina en la Bluefields Indian and Caribbean University BICU, actualmente no existe ninguna investigación que permita aportar información sobre la situación actual de la escuela. De esta manera, este estudio bridará información para conocer de fuentes primarias, información permita que las autoridades logren incidir y dar respuestas positivas a las debilidades, enfrentar las amenazas y aprovechar las fortalezas y oportunidades con que cuenta la escuela de medicina.

La Escuela de Medicina de la BICU, surge ante un análisis de las necesidades de la población caribeña en el ámbito de la salud, tales como: incremento de diferentes enfermedades epidemiológicas, limitadas oportunidades que presentan los estudiantes indígenas $y$ afro descendientes en calificar a la carrera de medicina en las universidades del pacifico, el reconocimiento de la desigualdad de oportunidades de los indígenas y afro descendientes. Así como la valoración del número de profesionales de la salud autóctonos existentes en la región del caribe, los problemas educativos, culturales económicos y psicológicos que enfrentan estos estudiantes indígenas y afro descendientes en las universidades del pacifico que inciden en los retiros de los estudiantes de las carreras a las que tienen oportunidad. (Cash 2008). La escuela presenta como misión formar profesionales de la salud, con criterios de excelencia, en el ámbito científico - técnico y humano, favoreciendo el desarrollo de sus competencias y adecuándolas a las necesidades e idiosincrasias de los pueblos indígenas, afro descendientes y mestizos de la Costa Caribe Nicaragüense, y su Visión: Ser una escuela líder en creencias de la salud intercultural en el ámbito nacional e internacional mediante educación innovadora y la investigación aplicada al bienestar del ser humano(Cash, Sánchez, Hodgson Wallace, aprobado en Consejo Universitario BICU 2008).

Las autoridades de la universidad expresan que con esta carrera, la Bluefields Indian Caribbean University (BICU), proyecta concretar su compromiso con las y los bachilleres de las Regiones Autónomas, ofreciendo la carrera de Medicina, permitiéndoles a éstos formarse para responder al sistema de salud como parte integrante de la realidad del medio que le rodea y en el que la diversidad lingüística y cultural es una realidad a tener en cuenta, respetar y atender, convirtiéndolos en seres humanos pensantes con capacidad de desarrollarse exitosamente en la sociedad en la que debe desenvolverse e impulsar el desarrollo de la misma. (Cash, Sánchez, Hodgson Wallace, aprobado en Consejo Universitario BICU 2008).

En el marco del fundamento filosófico de la universidad, se propone formar médicos profesionales comprometidos con los elementos científicos y humanísticos para llevar al ser humano a preservar, mantener y recobrar su salud en los distintos estadios. Por tanto, ofrece al educando una base filosófica de vida personal y profesional basados en valores éticos y morales; considerando el proceso de enseñanza aprendizaje como un proceso en el cual se comparten los conocimientos. (Cash, Sánchez, Hodgson Wallace, aprobado en Consejo Universitario BICU 2008). 
El objetivo principal de esta investigación fue determinar factores (fortalezas, oportunidades, debilidades, amenaza y clima organizacional (liderazgo, motivación, reciprocidad y participación), que inciden en la conducción de la escuela de medicina de la Bluefields Indian and Caribbean University BICU en el primer trimestre 2017, desde la percepción de los administrativos y docentes de la escuela para responder a la siguiente interrogante ¿Cuáles son los factores (fortalezas, oportunidades, debilidades, amenazas, y clima organizacional (liderazgo, motivación, reciprocidad y participación) que inciden en la conducción de la escuela de medicina de la Bluefields Indian and Caribbean University BICU en el primer trimestre del 2017? . Los hallazgos permitieron tener una visión general de la situación de la escuela de medicina, generar propuestas que sean consideradas e implementadas por las autoridades institucionales.

La presente investigación no experimental se trabajó bajo el enfoque cualitativo, el cual "busca describir, comprender e interpretar los fenómenos, a través de las percepciones y significados producidos por las experiencias de los participantes " (Hernández, Fernández y Baptista 2010:11) utilizando el método de investigación observacional (Piura, 2006), con un nivel inicial del conocimiento, es explorativo (Piura, 2006), de acuerdo al tiempo de ocurrencia es retrospectivo, Hernández, Fernández y Baptista 2014. Según el período y secuencia es transversal, Hernández, Fernández y Baptista 2014. Los instrumentos para la recolección de la información fueron: entrevistas abiertas y cuestionario propuesto por el programa subregional para el desarrollo de la capacidades gerenciales en los servicios de salud Centroamericana y Panamá que consta de 80 ítem en una escala Likert de cinco puntos.

Brunet (1987), afirma que el clima organizacional constituye la configuración de las características de una organización, así como las características personales de un individuo pueden constituir su personalidad. El clima dentro de una organización es un componente multidimensional de elementos; por tanto, puede descomponerse en términos de estructuras organizacionales, tamaño de la organización, modos de comunicación, estilo de liderazgo de la dirección, etc. Todos estos elementos se suman para formar un clima particular dotado de sus propias características que representa, en cierto modo, la personalidad de una organización e influye en el comportamiento de las personas en cuestión.

Alvarez (2001), Segredo y Reyes (2004) y Méndez (2006), definen el clima organizacional como el conjunto de caracteristicas predominantes en el ambiente interno en que opera la organización, las cuales ejercen influencia sonbre las conductas, creencias valores y percepciones de las personas, determinando su comportamiento y sus niveles de satisfaccion.

Alvarez et al, (2009) mencionan que detectar las necesidades de los usuarios, de los trabajadores y de la institucion en general es determinante para el logro de los objetivos organizaciones y representa un area de oportunidad para aqellas tecnicas y procedimientossusceptibles de mejora. Por ello, se destaca como requisito indispensable para el desarrollo y bienestar de los centros medicos realizar evaluacines peridicas que permitan determinar las percpciones que tanto los usuarios como los propios empleados tienen del proceso y de las condiciones enque se prestan los servicios de salud,conel objetivo fundamental de identificar los elementos que contribuyen u obtaculizan el cmplimiento de las metas institucionales (Senic y Marinkovic, 2012; Sant y sampedro, 2012).

Sandoval (2004) y Garcia,Moro y Medina (2010) mencionana que el clima organizacinal es definido por una serie de caracteristicas objetivas y relativamene perdurables en la organizacin y que peden intervenir en aspectos laborales, tales como el comportamiento inividual, los estilos de direccion, las normas, politicas 
y de manera general, en el sistema orgaizacioanalde la institucion.

Pérez de Maldonado, Maldonado y Bustamante (2006) afirman que estudiar el clima organizacional permite tomar en cuenta las percepciones de los trabajadores, a fin de que la alta dirección tenga una opinión compartida de la atmosfera de trabajo en la cual se realizan las actividades de gestión, y con base en ello diseñar planes de acción que favorezcan el desempeño y compromiso de los empleados.

Rensis Likert, propuso en forma pionera el uso de encuestas para hacer diagnósticos de las organizaciones. La teoría de Likert supone que las prácticas de gestión participativa llevan a niveles más elevados de desempeño organizacional. Siguiendo esta teoría, se utilizaron encuestas para captar datos de las percepciones de los empleados acerca de una variedad de prácticas de gestión organizacional, como el liderazgo, la comunicación y la toma de decisiones.

Mauro (1995) Las normas culturales y las costumbres establecidas incluyen los hábitos, las maneras de pensar, los valores y las normas informales no escritas de una sociedad. Estas fuerzas socioculturales funcionan en los ámbitos local, nacional y regional, y tienen una profunda influencia en la manera en que las organizaciones realizan sus actividades y en lo que valoran en términos de productos y efectos. Considero que esta situación ha estado afectando a la escuela de medicina para llevar una conducción más efectiva.

Chruden y Sherman consideran que toda organización posee su propia y exclusiva personalidad o clima que la diferencia de otras. Sostienen que la gerencia debe prestar mucha atención a este aspecto, ya que entender el clima de la organización conlleva al logro de los objetivos establecidos. Brow y Moberg, por su parte, manifiestan que el clima se refiere a una serie de características del medio ambiente interno organizacional tal y como lo perciben los miembros de esta.
Segredo, realizó una investigación con el objetivo de identificar la percepción de los directores de áreas de salud sobre el clima organizacional de sus instituciones en Cuba, donde estudió cuatro dimensiones básicas: motivación, liderazgo, reciprocidad y participación, obteniendo como resultado que el clima organizacional que perciben los directores de áreas en el lugar que se desempeñan no es totalmente adecuado, con dimensiones afectadas como la motivación y la reciprocidad.

\section{Enfoque Sistémico para el Análisis de la Efectividad Grupal y Organizacional}

De acuerdo con este enfoque, la efectividad de un grupo y de una organización, depende de numerosos factores que se encuentran estrechamente interrelacionados, y entre los cuales existen relaciones de dependencia mutua. De manera que al hablar de enfoque sistémico nos estamos refiriendo a la orientación que propugna la idea de visualizar al grupo u organización como totalidades orgánicas e íntegras de partes interrelacionadas e interdependientes. Cada parte (subsistema) y proceso dentro de la totalidad es afectada y tiene impactos en las restantes. Por ello, la modificación de cualquiera de los componentes afecta a los restantes.

\section{Para explicar los factores que inciden en la escuela de medicina definimos las variables que sustentan este estudio:}

Rafael Muñiz, FODA, es una metodología de estudio de la situación competitiva de una empresa en su mercado y de las características internas de la misma, a efectos de determinar sus Fortalezas, Oportunidades, Debilidades y Amenazas; las debilidades y fortalezas son internas a la empresa; las amenazas y oportunidades son externas, y se presentan en su entorno. Según Ferecheva, FODA, es considerada una técnica fue originalmente propuesta por Albert Humphrey durante los años 60's y 70’s en los Estados Unidos. 


\section{Dimensiones y categorías que componen el instrumento Inventario del Clima Organizacional, según la OMS y OPS.}

En este estudio se analizaran cuatro dimensiones: el liderazgo, la motivación, la reciprocidad o compromiso y la participación. A su vez, a cada dimensión corresponden 4 categorías. Para el análisis del liderazgo se consideran las siguientes categorías: dirección, estímulo de la excelencia, estímulo del trabajo en equipo y solución de conflictos.

\section{Liderazgo}

a. La dirección proporciona el sentido de orientación de las actividades de una unidad de trabajo, estableciendo los objetivos e identificando, en forma clara, las metas que se deben alcanzar y los medios para lograrlo. En ese proceso, se determina y cuantifica la responsabilidad de cada subalterno y las interacciones con otros individuos o grupos para lograr resultados esperados; por ello se hace necesario proporcionar suficiente información, así como la orientación técnica para poder tomar decisiones.

b. El estímulo de la excelencia significa poner énfasis en la búsqueda de mejorar constantemente, mediante la incorporación de nuevos conocimientos e instrumentos técnicos. Se promueve dentro de un ambiente en donde la jefatura demuestra una preocupación genuina por el desarrollo de las personas; las exhorta y apoya para que actualicen sus conocimientos y las impulsa constantemente a mejorar los resultados.

c. El estímulo del trabajo en equipo. La gestión organizacional se basa en el trabajo en equipo, al interior y entre las unidades administrativas, buscando fundamentalmente el logro de objetivos comunes. La participación organizada es el elemento indispensable en el trabajo en equipo, la cual tiene cabida siempre y cuando se estructure un ambiente de apoyo mutuo.

d. La solución de conflictos, está considerado como parte inherente en la vida institucional. En la organización siempre surgen conflictos que se originan por diversas causas, aunque en definitiva tienen un trasfondo común: son diferencias de percepciones e intereses que compiten sobre una misma realidad.

\section{Motivación}

a. Realización personal. El objetivo de cualquier ser humano es el de su realización, lo que trasciende los límites de la expresión individual por su dimensión social, y rebasa también los límites del momento por su dimensión histórica. La realización integral del hombre solo puede llevarse a cabo dentro de un contexto ocupacional en el cual es posible que la persona aplique sus habilidades. El hombre habrá de conocer todas las oportunidades de autorrealización que tiene con su trabajo y tomar conciencia de la trascendencia comunitaria e histórica de su aportación en la organización. Por eso la naturaleza del trabajo es especialmente importante, pues para que represente oportunidades de realización personal, el trabajo debe permitir el control del destino propio, libertad de expresión y oportunidades para la experimentación. Si estas necesidades de realización personal son satisfechas, el individuo se motiva a canalizar hacia el trabajo sus capacidades más creativas y constructivas. Cuando las satisfacciones en el desempeño del trabajo superan las expectativas de los empleados, estos se convierten en aliados de la institución.

b. Reconocimiento de la aportación. Cuando la organización reconoce el esfuerzo realizado por cada persona o grupo en la ejecución de las tareas asignadas para el logro de los objetivos institucionales, posee un alto potencial motivador, 
porque satisface las necesidades de realce del ego. Todo individuo tiene necesidades de alcanzar una condición especial dentro de la organización, que satisface cuando se le da reconocimiento y oportunidad para demostrar su capacidad. Aquellas organizaciones que satisfacen estas necesidades de reconocimiento como forma de motivación, tienden a poner énfasis en la recompensa pública y en los sistemas de reconocimiento del mérito de los funcionarios a partir de sus aportaciones para el logro de los objetivos.

c. Rresponsabilidad, que es la capacidad de las personas para responder por sus deberes y por las consecuencias de sus actos. Toda actuación del hombre tiene una proyección comunitaria y es en el trabajo organizado donde sus actos adquieren significado. Para que las personas puedan responsabilizarse de sus acciones, necesitan conocer y comprender su ubicación y su proyección dentro de la organización. Cuanto mayor es el grado de comprensión que las personas tienen de la labor que realizan y de la proyección social de esta, su capacidad por adquirir responsabilidad es más alta. La responsabilidad en una organización se mide por el grado de compromiso con que los trabajadores deciden asumir, consciente y voluntariamente, sus deberes en respuesta a las demandas de trabajo. Para que exista responsabilidad, el trabajador, además de tener un sentido de identificación con los objetivos que persigue la institución, debe contar con la confianza por parte de los superiores, lo cual incrementa la motivación personal.

\section{d. La adecuación de las condiciones de trabajo es} otra categoría de análisis de la motivación. Las condiciones ambientales físicas y sicosociales en que se realiza el trabajo, así como la calidad y cantidad de los recursos que se suministran para el cumplimiento de las funciones asignadas, deben ser congruentes con la naturaleza misma del trabajo. Cuando la adecuación de estas condiciones no se da, se produce insatisfacción en el trabajador, repercutiendo en bajos niveles de desempeño y en la calidad de los servicios producidos.

\section{Reciprocidad}

a. Aplicación al trabajo. Cuando un individuo se encuentra identificado plenamente con su trabajo y con la institución, adopta comportamientos que superan los compromisos del contrato formal, manifestados en la dedicación por el logro de los objetivos, que lo impulsa a asumir responsabilidades y desplegar todo su ingenio y su creatividad en la solución de los problemas, adquiriendo hábitos de trabajo donde priman la exactitud, el esmero y el orden.

b. El cuidado del patrimonio institucional es la segunda categoría de análisis y se refleja por medio del cuidado que los funcionarios dan a los bienes materiales de la institución, así como también en su preocupación por el fortalecimiento y defensa de los valores e imagen de la misma. La organización pretende concientizar a sus empleados para que adopten una actitud de aprecio y defensa respecto a los recursos asignados, espera cuidado y buen uso de los equipos materiales, y de las instalaciones a su cargo, sean estos de su puesto de trabajo, de una unidad o de la organización en general, contribuyendo a eliminar el despilfarro y la imagen desagradable en las dependencias físicas. En el patrimonio institucional se incluye también el prestigio de la institución, cuya apreciación social depende en gran parte del trato que los empleados dan a las personas que acuden en busca de los servicios, el cual debe estar basado en principios elementales de calidad de la atención, respeto y cortesía.

c. Retribución. La organización habrá de optimizar los contenidos retributivos de las relaciones laborales en beneficio de sus recursos humanos, 
para contribuir a su realización personal y desarrollo social y en respuesta a las aportaciones de las personas con su trabajo. Los principales mecanismos retributivos, que la organización pone a disposición de sus miembros pueden resumirse en: sistema justo y competitivo de remuneración; prestaciones compatibles con las posibilidades económicofinancieras de la institución en el contexto de las políticas salariales nacionales; sistemas de promoción, con reconocimiento al desempeño, que brindan la oportunidad a los individuos de hacer carrera dentro de la organización; sistemas de capacitación y desarrollo de los recursos humanos, para incrementar conocimientos y habilidades de las personas en referencia al perfil ocupacional; sistemas o programas de bienestar social para favorecer la integración de los empleados y sus familias, y permitir la satisfacción de sus necesidades culturales y recreativas; además de estabilidad laboral que elimina la incertidumbre y la ansiedad del trabajador frente al futuro.

d. Equidad. Los trabajadores esperan tener acceso a las retribuciones, indicadas anteriormente, por medio de un sistema equitativo que trate a todos los funcionarios, en condiciones de igualdad de trabajo, con iguales beneficios. Especialmente aspiran a ser tratados con imparcialidad en procesos de selección y promoción, solamente basados en la competencia y el mérito frente a los requisitos exigidos.

\section{Participación}

a. Compromiso con la productividad. Este se da en la medida en que cada individuo y unidad de la organización, en armonía con los demás componentes, realiza con óptima eficacia y eficiencia el servicio que le corresponde, mediante el cumplimiento de las funciones conforme a estándares preestablecidos. b. Compatibilización de intereses. La función básica del proceso de conducción de la participación en la organización es, sin duda, integrar la diversidad de sus componentes en una sola dirección; la del cumplimiento de los objetivos institucionales. Los retos principales para lograr la participación organizada se refiere a la compatibilización de intereses en las siguientes áreas de conflicto: 1) competencia para obtener recursos limitados, por lo que se debe establecer una jerarquización de prioridades en función de los objetivos institucionales; 2) distribución del poder: el acceso a los mecanismos de información, como medios de poder, se convierten con frecuencia en metas particulares de individuos y unidades, que distraen sus esfuerzos en la disputa, en detrimento de las posibilidades de hacer aportaciones constructivas para el logro de los objetivos institucionales; 3) tendencias a la autonomía: toda organización tiene la necesidad de asegurar que sus miembros respeten ciertas normas de comportamiento, sin excluir la flexibilidad necesaria para preservar la capacidad de adecuación constante ante las condiciones variantes que la afectan.

c. Intercambio de información. Para obtener la acción coordinada entre personas y grupos, es necesario comunicarse e intercambiar información sobre los propósitos comunes y los medios que cada uno posee para contribuir al logro de ellos. La libre circulación de información, entre diferentes unidades de trabajo, es el mecanismo que hace que los grupos mantengan vínculos de cooperación. La existencia del flujo de información entre los grupos es fundamental; sin embargo, es preciso cuidar de su calidad, pues si esta es tergiversada o malintencionada, generará un proceso de disociación en lugar de participación organizada.

d. Involucración en el cambio. Las organizaciones están constantemente dinamizadas por múltiples procesos de cambio, profundos y acelerados, que 
permanentemente plantean al hombre nuevas interrogantes. Ante esta realidad el hombre no permanece impasible; siempre adopta una postura. La actitud comprometida, orientando y promoviendo el cambio, construyendo el futuro a partir de la experiencia asimilada en el pasado, es la verdadera respuesta ante el proceso de cambio. El hombre no debe ignorar el cambio ni temerle, no puede oponerse a él, sino que ha de asumirlo porque lo comprende, lo canaliza por la orientación que él mismo le da, y lo promueve estimulando las condiciones favorables.

\section{MATERIALES Y MÉTODOS}

El presente estudio se llevó a cabo en el campus de la Bluefields Indian and Caribbean University BICU, específicamente en la Escuela de Medicina. Se fundamenta en la integración sistémica los métodos y técnicascualitativas(Hernandez,Fernández,\&Baptista) y recolecta de informacion a través de entrevista abierta utilizando la técnica FODA y cuestionario con cuatro dimensiones: liderazgo, motivación, reciprocidad o compromiso y participación.

El universo del estudio estuvo compuesto por tres docentes del área administrativa y 45 docentes horarios que imparten clase en la carrera de medicina. La muestra en el ámbito cualitativo de acuerdo con Ary, Jacobs y Razavieh (2004), "Se recomienda seleccionar de un $10 \%$ a un 20\% de la población accesible" (p. 141). Estos autores señalan que "la Consideración más importante al sacar una muestra es su representatividad" (p.141). Por tanto, la muestra no probabilística, estuvo conformada por el $25 \%$ del universo: dos personas del área administrativa y 10 docentes. Para la selección de los participantes se utilizó el muestreo por conveniencia (Las personas que tenían más años de trabajar con la escuela de medicina). Esta información fue proporcionada por la directora de la escuela.

\section{Técnica e instrumentos de recolección de datos}

Las técnicas de recolección de datos que se aplicaron, según los objetivos específicos de la investigación, son elFODA a través de entrevistas directas y el cuestionario donde los participantes responden a un cuestionario considerado como una prueba proyectiva, que permite identificar las percepciones individuales y grupales sobre la organización, que inciden en las motivaciones y el comportamiento de las personas y está diseñado con base a un conjunto de 80 ítem en forma de escala de Likert cinco respuestas, diligenciadas en forma confidencial por los funcionarios, emitiendo los juicios que, a su modo de percibir la realidad, caracterizan la organización.

\section{Validez y confiabilidad del instrumento}

\section{Estadísticas de fiabilidad}

\begin{tabular}{ll}
\hline Alfa de Cronbach & N de elementos \\
\hline .829 & 80 \\
\hline
\end{tabular}

Se validó el cuestionario a través de la aplicación del índice de Alfa de Cronbach obteniendo un resultado de confiabilidad de 0.829 . Esta prueba se realizó con otro grupo de docentes que no pertenecen a la escuela de medicina.

Una vez realizada las entrevistas, se procesaron y analizaron agrupando la información de acuerdo a los indicadores: fortaleza, oportunidades, debilidades y amenaza.

En la aplicación del cuestionario se garantizó el anonimato y no se recogieron datos personales. Este cuestionario se calificó de la siguiente manera: según la escala de Likert, el protagonista marcaria con una $\mathrm{X}$ la respuesta considerada por él o ella. Posteriormente se agruparían en dos categorías donde 1 corresponde a bueno, muy bueno, excelente y 2 a regular y deficiente. Toda la información obtenida fue vaciada en una base de datos para su procesamiento a través del paquete 
estadístico SPSS (Statistical Package For Social Sciences) para Windows en su versión 21.

\section{RESULTADO DISCUSION}

Los resultados presentados en este escrito, corresponde al análisis de datos cualitativos técnica FODA y posteriormente, los resultados de aplicación de cuestionario de frecuencias de las respuestas. Este análisis permitirá describir el grado general de percepción de los participantes respecto a la conducción de la escuela.

Mediante el análisis de los resultados obtenidos a través de entrevistas aplicadas a administrativos y docentes de la escuela, se identificaron las principales Fortalezas, Oportunidades, Debilidades y Amenazas de la escuela.

\section{Fortalezas en la conducción de la escuela de medicina}

Los principales hallazgos, muestran las fortalezas identificadas en con la metodología FODA utilizada, entre las que se destacan:

- Interés de las autoridades de la universidad, en mejorar las situaciones presentadas para elevar los indicadores de desarrollo científicos de la universidad.

- Ser la única escuela de medicina en la Región Autónoma de la Costa Caribe Sur que forma estudiantes en contextos multiculturales.

- El 98\% del personal que labora en la escuela de medicina son altamente calificados especialistas. Docentes con disponibilidad para la enseñanza.

- Los estudiantes de la carrera de medicina tienen reconocimiento a nivel nacional al haber obtenido primeros lugares en concurso.

- Las secciones de medicina contienen equipo didáctico como data show.

- Docentes y estudiantes han recibido capacitaciones del paquete informático EpiInfu y como ingresar a la plataforma virtual, a las bibliotecas virtuales. En la universidad hay WF.

\section{Oportunidades en la conducción de la escuela de medicina}

- El apoyo de la Organización Panamericana de la Salud.

- Coordinación con la Facultad de Medicina de la Universidad UNAN- Managua y UNAN-León.

- Convenio establecido con el Ministerio de salud.

- Expresiones positivas de pobladores de los servicios brindados por los estudiantes de la BICU.

\section{Debilidades en la conducción de la escuela de medicina}

- Operatividad de la escuela con limitado personal, duplica el trabajo del personal administrativo.

- El 100\% del claustro de docentes que imparten clase son contratados como horario.

- Los recursos humanos y materiales son insuficientes para estimular la investigación científica.

- Poca participación de los docentes en las capacitaciones.

- Poco reconocimiento social al docente.

- Poco acompañamiento, seguimiento y monitoreo de las prácticas de campo que realizan los estudiantes.

- Falta de presupuesto para equipar los laboratorios y brindarles mantenimiento.

- Reducido espacio en la oficina de medicina.

- Falta programa de reforzamiento para los estudiantes que presentan problemas en su rendimiento académico.

- Curricular académica no se ajusta a contextos comunitarios e interculturales.

- Endeble sistema de comunicación con los docentes y estudiantes.

- Disconformidades existentes en la oficina de medicina.

- Poca asignación de secciones para la carrera de medicina. 
- El laboratorio de computación, las máquinas están obsoletas y no funcionan.

- El laboratorio de maqueta utilizado en las prácticas de los estudiantes este se encuentra descuidado, hacen falta piezas y otras maquetas de utilidad.

- Pocas evaluaciones y seguimiento a la escuela de medicina, está trabajando muy coyunturalmente ajustándose a las necesidades.

- Hacen falta seis recursos humanos calificados para funcionar con el 100\% del personal.

Amenazas en la conducción de la escuela de medicina

- Carga laboral que desarrolla el personal administrativo:tareasasistenciales yadministrativas afectan el cumplimiento de las supervisiones y monitoreo de los docentes y estudiantes en aras prácticas.

- El 100\% del claustro de docentes que imparten clase son contratados como horario.

- Alto precios de los materiales requeridos por la escuela de medicina.

- Falta un laboratorio de necrosis.

- Equipar los laboratorios existentes.

De acuerdo al cuestionario sobre clima organizacional, aplicados a administrativos $\mathbf{y}$ docentes que laboran en la escuela de medicina se clasifican en relación a las categorías 1. (Excelente, muy bueno, bueno), 2 (regular y deficiente).

En el grafico No. 1 se puede observar que la dimensión liderazgo, de forma general clasificó como clima organizacional regular, al comparar por variables se aprecia que la variable estímulos de trabajo obtiene la puntuación más alta, clasificándola como muy buena. Las las variables solución de conflicto, estímulos de la excelencia y dirección clasifican en un nivel de regular.

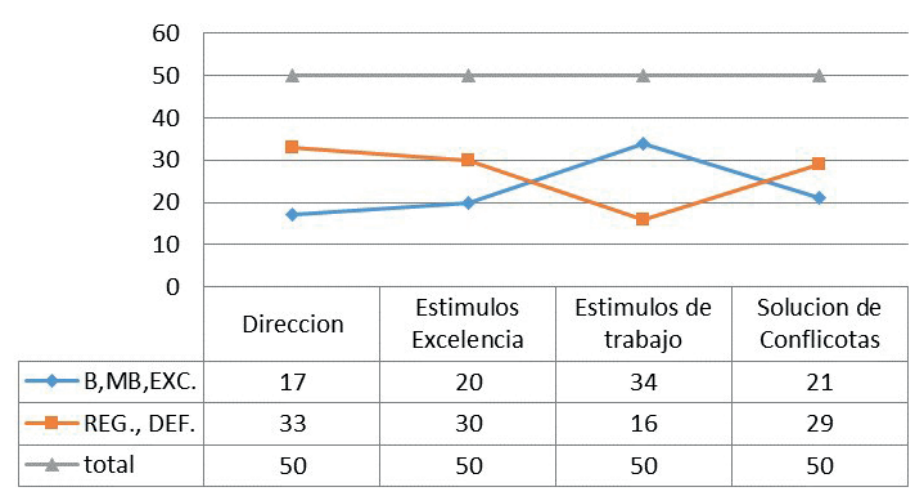

Gráfico 1. Liderazgo

El grafico No. 2 se puede observar que la dimensión motivación, de forma general clasificó como clima organizacional regular, al comparar por variables se aprecia que las variables realización personal y responsabilidad obtiene la puntuación más alta, clasificándola como muy buena, las variables reconocimiento y condiciones de trabajo se clasifican en un nivel de regular.

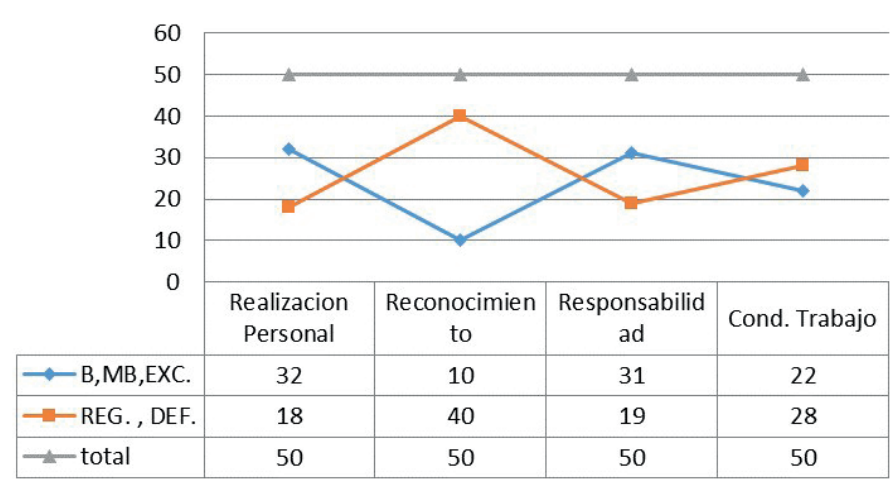

Gráfico 2. Motivación

El grafico No. 3 se puede observar que la dimensión reciprocidad, de forma general clasifico como clima organizacional bueno, al comparar por variables se aprecia que las variables cuidado del patrimonio y aplicación al trabajo, obtiene la puntuación más alta, clasificándola como muy buena, las variables retribuciones y equidad se clasifican en un nivel de regular. 


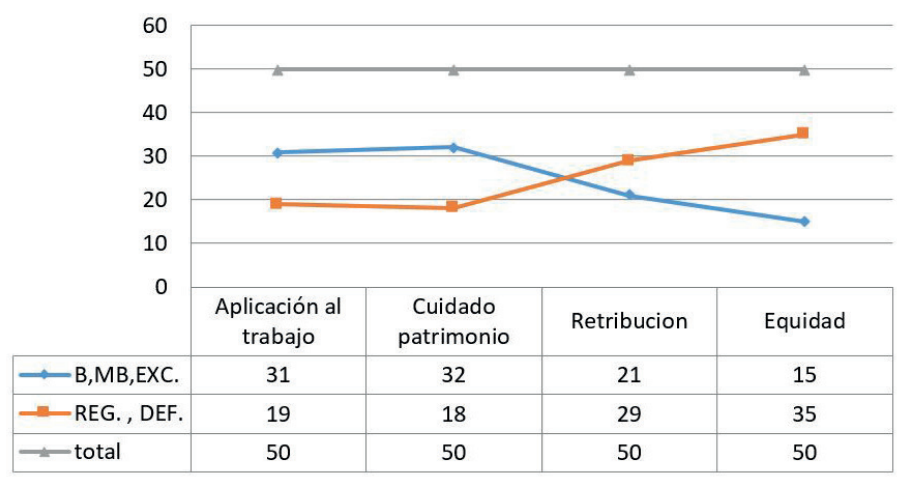

Gráfico 3. Reciprocidad

El grafico No. 4 se puede observar que la dimensión participación, de forma general clasificó como clima organizacional regular, al comparar por variables se aprecia que la variable compatibilización obtiene la puntuación más alta clasificándola como buena, las variables compromiso con la productividad, intercambio de información e involucramiento al cambio se clasifican en un nivel de regular.

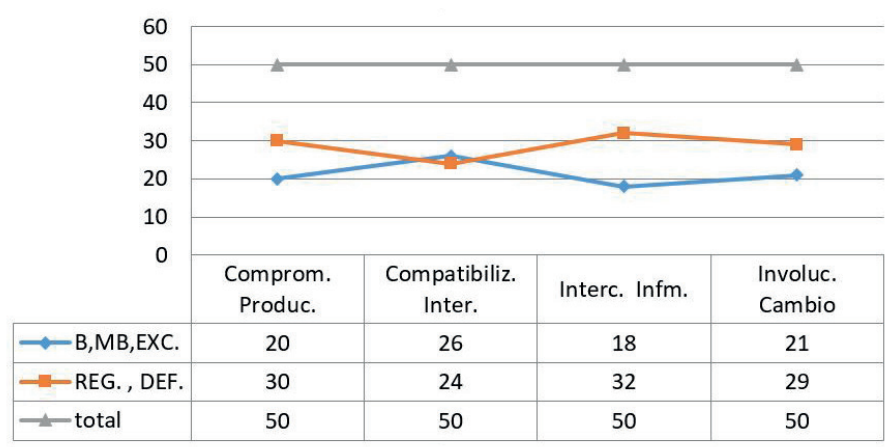

\section{Gráfico 4. Participación}

Análisis del grafico No. 5 donde se representan las 16 variables estudiados en base a lo establecido en programa subregional para el desarrollo de la capacidades gerenciales en los servicios de salud Centroamericana y Panamá.

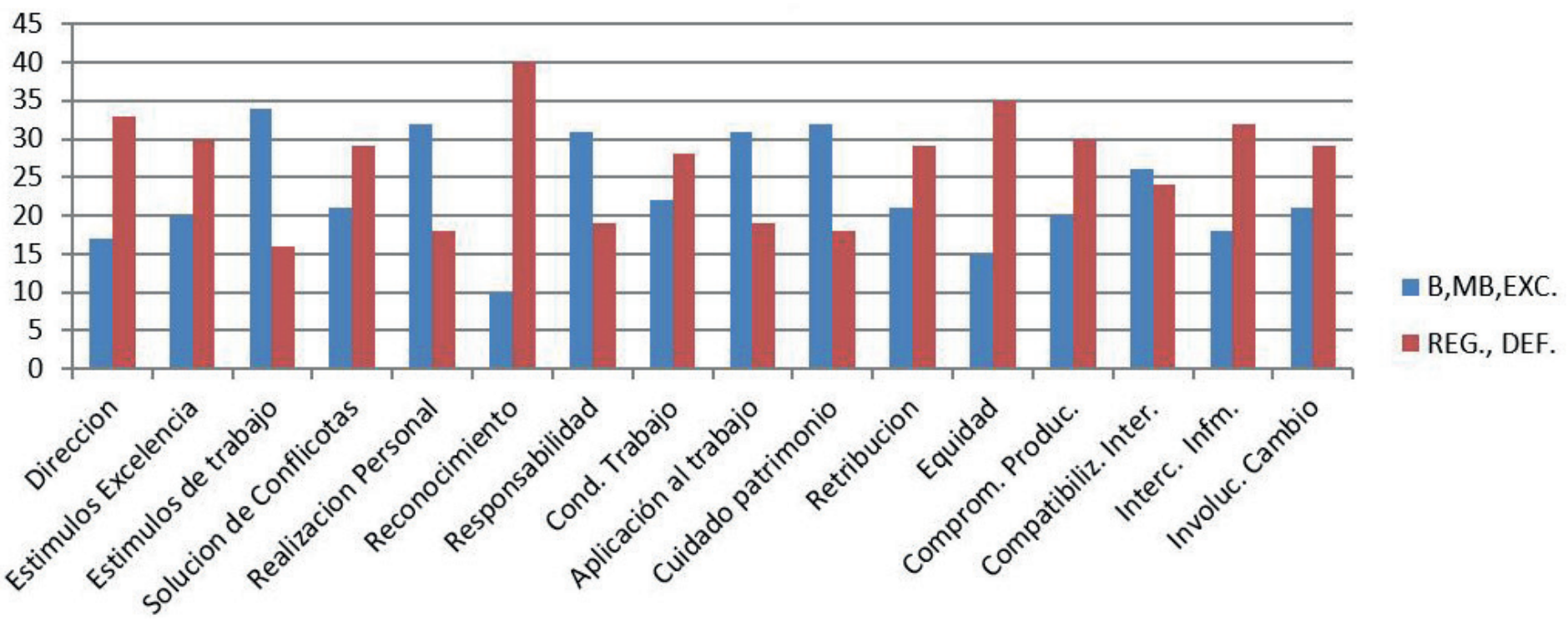

Gráfico 5. Parámetros evaluados

Tomando como fortaleza las variables que clasificar entre buenas, muy buenas y excelente. En relación a estimulo de trabajo se refiere a la gestión organizacional que se basa en el trabajo en equipo, al interior de las unidades y entre las unidades administrativas, buscando fundamentalmente el logro de objetivos comunes. Cuidado del patrimonio, se refiere al cuido que los funcionarios exhiben de los bienes y las cosas materiales de la institución, así como también por su preocupación por el fortalecimiento y defensa del prestigio de su repartición y de los valores y la imagen de la institución. Realización personal, basada en el objetivo globalizante de cualquier ser humano es el de su realización integral, lo que trasciende los límites de la expresión individual por su dimensión social y rebasa también los límites del momento por su dimensión 
histórica. La responsabilidad, es la capacidad de las personas para responder por sus deberes y por las consecuencias de sus actos. En relación a la Aplicación al trabajo, está referido a cuando un individuo se encuentra identificado plenamente con su trabajo y con la institución adopta comportamientos, que de alguna manera superan los compromisos que se derivan del contrato formal de empleo; manifestándolos en la dedicación por el logro de los objetivos de su unidad y de la institución.

Tomando como debilidades las variables que clasificaron como regular a deficiente. En relación al reconocimiento, la Escuela de Medicina, no está reconociendo ni da crédito al esfuerzo realizado por cada persona o grupo en la ejecución de las tareas asignadas para el logro de los objetivos institucionales, le hace falta aprovechar el alto potencial motivador del personal docente. La Equidad, se percibe a la escuela con debilidades en el sistema equitativo que trate a todos los funcionarios, en condiciones de igualdad de trabajo, con iguales beneficios. La Dirección, en la escuela se reflejan debilidades en proporcionar el sentido de orientación de las actividades de una unidad de trabajo, estableciendo los objetivos perseguidos e identificado, en forma clara, las metas que se deben alcanzar y los medios para lograrlo. En Intercambio de información, los resultados muestran que es necesario comunicarse e intercambiar información importante sobre los propósitos comunes y los medios que cada uno posee para contribuir al logro de ellos. El Estímulo a la excelencia, refleja debilidades en la gestión organizacional se basa en el trabajo en equipo, al interior de las unidades y entre las unidades administrativas, buscando fundamentalmente el logro de objetivos comunes. Compromiso con la productividad, en este caso, los protagonistas de este estudio reflejan la falta de unidad de la organización, en armonía con los demás componentes, realiza con óptima eficacia y eficiencia el servicio que le corresponde, mediante el cumplimiento de las funciones individuales y de las reparticiones conforme a estándares de calidad y cantidad preestablecidas y adoptadas.
La teoría de Herzberg F. (1960), teoria de los dos factores, afirma que las personas estan impulsadas por los motivadores más que por factores de mantenimintos: factores extrinsecos (la remuneracion, la seguridad laboral, y el titul del puesto, condicines de trabajo, prestaciones y relaciones), y los factores intrinsecos (el trabajo mismo, el logro, el reconocimiento, el desafio y el desarrollo profesional. En el estudio se refleja bastante bien los factores intrinsecos lo que hay que estimular los factores extrinsecos.

El grafico No. 6 se puede observar el análisis factor percepción de las dimensión, de forma general clasifico como clima organizacional regular, al comparar por dimensiones se aprecia que la dimensión reciprocidad se encuentra con una discrepancia entre lo regular a bueno, la dimensión motivacional existe una diferencia entre regular a buena, mientras que las dimensiones: liderazgo y participación se clasifican de regular.

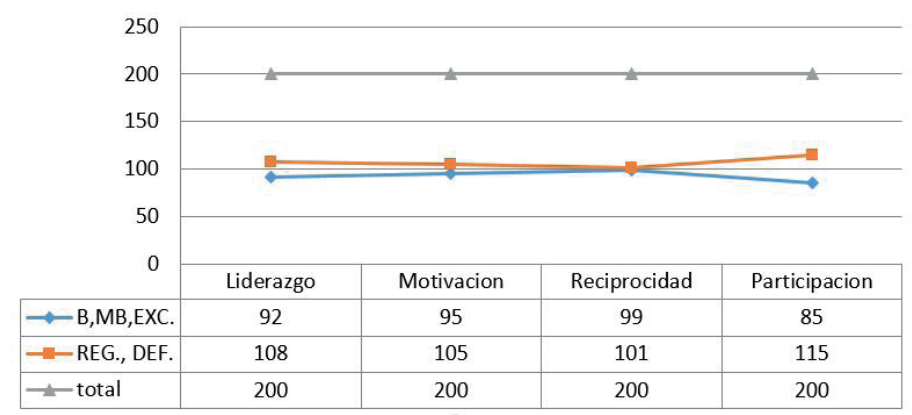

Gráfico 6. Análisis del factor percepción

En estudio realizado por Segredo Pérez, también se encontró que la percepción del clima organizacional presentaba, resultados no es totalmente adecuado, con las dimensiones afectadas como motivación y la reciprocidad. En este estudio se observa que las dimensiones de mayor afectación son: participación y liderazgo. No existe grado de coincidencia ente este estudio y el referido por Segrado.

Márquez (2001) en su estudio sobre servicio de salud, aplicando este modelo propuesto para el diagnóstico, obtuvo los resultados las categorías funcionamiento y 
estímulo al desarrollo organizacional en promedio muy bajos por lo que se encuentra en riesgo la contribución de las personas y los grupos formales al logro de los objetivos de la organización.

Los resultados de este estudio, reflejan la categoría estímulo al trabajo en un promedio muy bueno, por lo que la escuela de medicina debe de aprovechar esta contribución de las personas y los grupos formales al logro de los objetivos de la institución. No existe grado de coincidencia ente este estudio y el referido por Márquez.

El grafico No. 7 se puede observar el análisis perfil global del clima organizacional la escuela de medicina. La tendencia de lo ideal (Máximo) se refleja en un nivel bajo. En relación a lo que se debe lograr para mantener un clima organizacional aplicable (Teórico), se refleja en un nivel bajo. En relación a la percepción de los docentes (Real) el clima organizacional se encuentra en buena posición. Las dimensiones: que se encuentran con alto grado de debilidades son: Participación y Liderazgo.

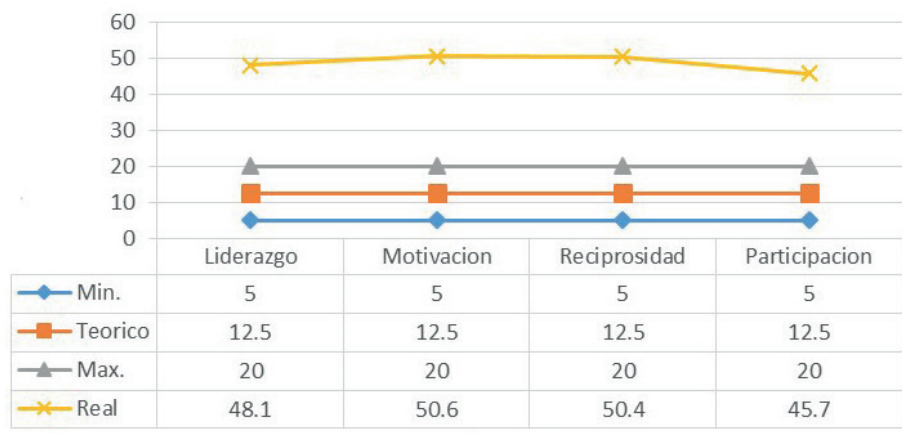

Gráfico 7. Análisis del perfil global del clima organizacional

Después de haber establecido el análisis FODA y Dimensiones de clima organizacional, las estrategias que se proponen son en base a sus debilidades para mejorar son las siguientes:

\section{Áreas críticas: Definición de funciones y estructura}

- Diseñar manual de funciones con un organigrama.

- Fortalecer el proceso de dirección.
- Diseñar mecanismos de control y evaluación.

- Diseñar sistema de comunicación interna y externas.

- Completar el personal requerido por la escuela

- Establecer un círculo técnico operativo de profesionales.

- Establecer programas de reforzamientos académico para los estudiantes.

\section{Áreas críticas: Clima organizacional}

\section{Alternativas de intervención:}

\section{Liderazgo:}

- Priorizaciones de tareas claves efectivas.

- Gerencia Administrativa del tiempo.

- Priorización de objetivos y metas a seguir.

- Métodos de confrontación para la solución de conflictos.

- Capacitación sobre mediación pedagógica.

- incidir en el sentido de orientación de las actividades de una unidad de trabajo, estableciendo los objetivos perseguidos e identificado, en forma clara, las metas que se deben alcanzar y los medios para lograrlo.

\section{Motivación:}

- Reconocimiento de estímulos para el personal.

- Mejoras a las condiciones físicas y psicológicas en que se labora.

- Calidad y cantidad de los recursos que se suministran para el cumplimiento de las funciones asignadas.

\section{Reciprocidad}

- Revisión y desarrollo de sistemas de retribución.

- Condiciones de igualdad de trabajo y beneficios.

- Equidad

- Participación: Mejoras del sistema de comunicación interna y externa.

- Análisis del campo de fuerzas restrictivas e impulsoras. 
- Ampliar comunicación e intercambiar información importante sobre los propósitos comunes y los medios que cada uno posee para contribuir al logro de ellos.

\section{CONCLUSIONES}

Al analizar la información arrojada de las entrevista para responder a la técnica FODA (fortalezas, oportunidades, debilidades y amenaza) y los resultados del cuestionario sobre clima organizacional (liderazgo, motivación, participación y reciprocidad), se infiere teóricamente que existe relación entre los resultados de ambas técnicas.

El análisis factor percepción de las dimensión, de forma general clasificó como clima organizacional a la escuela de medicina en la categoría de regular. Sin embargo, refleja las categorías que la escuela de medicina debe de aprovechar como contribuciones de las personas y los grupos formales para el logro de los objetivos de la institución, tales como: estímulo al trabajo, cuidado patrimonio, realización personal, responsabilidad y aplicación al trabajo. Así mismo, reflejan las categorías que debe tomar en cuenta para mejora: falta de reconocimiento, equidad, dirección, intercambio de información, estímulo a la excelencia y compromiso con la productividad.

La escuela de medicina debe ajustarse a los cambios generados por el transcurso de los años desempeñar mayor gestión organizacional, completar su equipo de trabajo, fortalecer sus recursos humanos, ampliar sus comunicaciones, proyectarse positivamente ante la sociedad, ser dinámica e integrativa, promover la realización de investigaciones educacional y de servicios de salud, transformarse en un nivel cualitativamente privilegiado, equipar sus laboratorios. La importancia de esta información se basa en la comprobación de que la información brindada por los protagonistas en las entrevistas sobre las fortalezas, oportunidades, debilidades y amenaza, logran coincidir con lo referido en el instrumento aplicado cuestionario sobre clima organizacional, esta información nos permite reflexionar sobre cómo está marchando la escuela, cual es la percepción de los integrantes, brinda las condiciones para motivar la gestión gerencial e incidir para mejorar.

\section{RECOMENDACIONES}

Dar a conocer a las autoridades la situación que está enfrentando la escuela de medicina permitirá sensibilizar e incidir en generar cambios que favorezcan el desempeño de las funciones.

Realizar evaluaciones sistemáticas de manera integral tanto a nivel interno como externa del desempeño y condiciones del clima organizacional de la escuela de medicina, ya que la escuela como institución formadora de recursos humanos debe de encontrarse con ambientes de calidad y eficiencia.

Implementar estrategias de comunicación efectivas que fortalezcan los aspectos de liderazgo y participación.

\section{AGRADECIMIENTO}

Al programa de Doctorado en Gestión y Calidad de la Investigación Científica, impulsado por la Universidad Nacional Autónoma de Nicaragua, Managua. Facultad Regional Multidisciplinaria de Estelí.

\section{BIBLIOGRAFÍA}

Álvarez (1992). "El constructo clima organizacional: conceptos, teorías, investigación y resultados relevantes" Revista interamericana de Psicología Ocupacional. Vo.11, No. 1 y 2.

Blauberg, I. (1977): "La Historia de la Ciencia y el Enfoque en Sistema". Rev. Ciencias Sociales, Academia de Ciencias de la URSS, (3) (29).

Bluefields Indian and Caribbean University (2013). Plan Estratégico Institucional 2014-2018. Bluefields, Nicaragua. 
Brunet L. (1987) El clima de trabajo en las organizaciones. $1^{\text {a }}$ ed. México: Editorial Trillas; 12.

Casales, J. y Rubí, A. (1986): "Desarrollo de una Metódica para la Descripción del Estilo de Liderazgo en Grupos Pequeños". Rev. Cubana de Psicología, Vol. 3, (3), págs. 3-32.

Castillo C, Del Pino N, Espinosa V. Cultura organizacional [Internet]; 2000 [citado 24 Ene 2012]. Disponible en: http://www.rrppnet.com.ar/ culturaorganizacional.htm.

Chruden HJ, Sherman J, Arthur W. Administración de personal. México, DF: CECSA; 1993.

Freddy E. Desempeño docente y clima organizacional en El Liceo "Agustín Codazzi”, de Maracay, Estado Aragua. Paradigma [Internet]. 1997 [citado 22 Ago. 2011]; 2. Disponible en: http://www. revistaparadigma.org.ve/

González G. (1994). “Organización de la atención", Centro de Investigación y Estudios de la Salud CIES, Managua septiembre.

Gonçalves AP. Dimensiones del clima organizacional [Internet]; 2001 [citado 23 Dic 2011]. Disponible en: http://www.calidad.org/articles/dec97/2dec97. htm.

Márquez Morales N. Estrategia de cambio en instituciones de atención primaria de salud. Rev. Cubana Med Gen Integ [Internet]. 2001 [citado 12 Nov 2011]; 17(6). Disponible en: http:/www.bvs. sld.cu/revistas/mgi/vol17_6_01/mgi1462001.htm.

OMS / OPS. Teoría y Técnicas de Desarrollo Organizacional. Programa Regional de desarrollo de servicios de salud. PSDCG-T-10. Vol.3. Módulo III. Unidad VI: 1-7; 1998.

Toro (1992). "Diseño y validación de un instrumento para evaluación del clima organizacional”. Revista
Interamericana de Psicología Ocupacional. Vol. 11 No. 1 y 2.

Rodríguez Salvá, Armando, Álvarez Pérez, Adolfo, Sosa Lorenzo, Irma, De Vos, Pol, Bonet Gorbea, Mariano H, \& Van der Stuyft, Patrick. (2010). Inventario del clima organizacional como una herramienta necesaria para evaluar la calidad del trabajo.RevistaCubanadeHigieney Epidemiología, 48(2), 177-196. Recuperado en 04 de julio de 2017, de http://scielo.sld.cu/scielo.php?script=sci arttext\&pid=S1561-30032010000200008\&lng=es \&tlng=es.

Sandoval Caraveo MC. Concepto y dimensiones del clima organizacional. Hitos de Ciencias Económico Administrativas. 2004; 27:78-82. Disponible en: http:/www.publicaciones.ujat.mx/publicaciones/ hitos/ediciones/27/08_ensayo_dimensiones.pdf.

Segredo PérezAM. Percepción del clima organizacional por directores de policlínicos. Cuba, 2003. Correo Científico Médico Holguín [Internet]. 2004 [citado 12 Abr 2012]; 8(4). Disponible en: http://www. cocmed.sld.cu/no84/n84ori7.htm.

Segredo Pérez AM, Pérez Perea L. El clima organizacional en el desarrollo de los sistemas organizativos. Rev. INFODIR [Internet]. 2007 [citado 12 Abr 2012]; 4. Disponible en: http:// www.sld.cu/galerias/doc/sitios/infodir/clima organizacional_en_el_desarrollo_de_sistemas organizativos.doc.

Segredo A, Reyes D. Clima organizacional en salud pública. Consideraciones generales. Correo Científico Médico de Holguín [Internet]. 2004 [citado 23 Ene 2011]; 8(3). Disponible en: http:// www.cocmed.sld.cu/no83/n83rev4.htm. 